\title{
Producción sobre movimiento social EN LAS REVISTAS DE CIENCIAS SOCIALES DE CHIAPAS: ALGUNOS COMENTARIOS
}

\author{
Sylvie Didou Aupetit
}

C omentar las investigaciones sobre movimiento social publicadas en Chiapas durante los últimos diez años, es decir, abordar fascinaciones y olvidos, no fue una tarea fácil por razones personales: no estoy al tanto de la historia de los grupos intelectuales y académicos regionales ni he leído sistemáticamente la numerosa producción regional, nacional e internacional sobre la región, en los últimos años. Además, no lo fue porque la noción misma de movimiento social plantea problemas de definición del objeto y de identificación de su inscripción disciplinar: ambas situaciones complicaron el proceso de selección de los documentos a examinar, a partir de una simple ojeada a los índices de las revistas. Como no era suficiente el tiempo para una lectura exhaustiva de los materiales y en ausencia de una preselección de los materiales por parte de ustedes, me estaré refiriendo tanto a un corpus incompleto como a un universo de referencia compartido con mis colegas.

Dicho esto y considerando a la vez mi condición de exterioridad, el repunte generalizado de las dis-

Sylvie Didou Aupetit, DIE-CINVESTAV. cusiones sobre ciencias sociales y humanas como disciplinas y la situación particular de Chiapas, decidí hacer un repaso rápido a los artículos no para evaluar contenidos sino para identificar cuáles habían sido los problemas sociales abordados y los acercamientos privilegiados para ello. Una primera lectura me dio la impresión de que parte de la producción académica es explicable por la presión de la coyuntura. Muchos textos, sobre todo los producidos en los últimos siete años, se legitiman en la urgencia de trabajos de campo que ayuden a comprender la génesis y las evoluciones de la movilización zapatista así como la participación de los indígenas en ésta. Y los discursos oscilan entre el análisis y la emoción, la descripción científica y la afectiva.

Por la multitud de miradas propias y ajenas que atrajo recientemente, Chiapas es un espacio original dentro del contexto nacional. Es también un laboratorio donde se ejemplifican las reiterativas preguntas sobre la construcción de los objetos de investigación, sobre su articulación con las condiciones sociales y políticas del entorno, sobre los procedimientos de implicación mutua de los actores y de los investigadores y sobre las interferencias entre lo político, lo ideológico y lo académico. 
Debido a ello, es imposible analizar la producción sobre movimiento social en Chiapas sin tomar en cuenta el zapatismo, en tanto acontecimiento a investigar y en tanto soporte de un campo emergente de producción académica, en el cual se busca desplegar las lógicas de la acción social y política o los motivos y formas de movilización de los actores o de los actuantes. Pero tampoco es factible estudiar la producción académica sin admitir que los anuarios o las revistas cumplen con necesidades de comunicación disciplinaria y de ubicación institucional/personal de las instituciones y, sobre todo, de los investigadores. Tienen por lo tanto un papel fundamental en la apropiación de los capitales personales y colectivos de prestigio intelectual. Aprovechando las libertades que otorga la distancia, quisiera referir las investigaciones sobre el juego social, contenidas en las revistas que me enviaron, a esas dimensiones.

\section{Producir desde fuera y desde dentro sobre zapatismo y sobre Chiapas en los noventa}

En la década pasada, el repunte de la producción editorial nacional e internacional sobre el movimiento social en Chiapas fue estrechamente dependiente del surgimiento del movimiento zapatista y tuvo lugar después de 1994. Aunque los libros y artículos aluden a diversos fenómenos, versan en su mayoría sobre aristas variadas de ese acontecimiento, conforme con acercamientos académicos o de circunstancia, con pretensiones de análisis o de divulgación.

El interés por el zapatismo nutrió el estudio de temas conexos, siempre referidos al tópico principal: por ejemplo, en la producción bibliográfica nacional e internacional sobre Chiapas, hay abundancia de artículos sobre mujeres (en lo relativo a formas de participación de las indígenas en el EZLN), sobre religión (en cuanto al papel de las iglesias en la constitución de bases de apoyo al EZLN y, principalmente, a la influencia de la teología de la liberación), sobre derecho y culturas indígenas, sobre la ubicación del EZLN en el marco de las organizaciones sociales y campesinas, sobre sus relaciones con los medios de comunicación, sobre los desplazamientos de población y sobre la problemática de la municipalización. En cambio, son escasas las aportaciones que se interesan por otras vertientes de la realidad chiapaneca, a no ser que sean especializadas en subcampos disciplinarios: por ejemplo, en las revistas de demografía, hay estudios sobre flujos migratorios desde Guatemala hasta Estados Unidos. En números temáticos, hay artículos sobre la Frontera Sur o bien aportaciones sueltas sobre temas de mayor amplitud, remitidos a la región como estudio de caso, no como centro de la reflexión.

En la producción extranjera sobre Chiapas, cabe diferenciar los artículos, que hablan en más de $80 \%$ sobre zapatismo, los libros, también dedicados en su mayoría al análisis de ese movimiento y las tesis: principalmente en las de doctorado, se encuentran algunos trabajos sobre educación (sindicalismo magisterial, educación indígena, demanda de servicios educativos), sobre salud pública, sobre producción agrícola, sobre neoliberalismo y reforma agraria y sobre la historia de Chiapas (historia regional, evangelización y protestantismo, asentamientos urbanos coloniales, principalmente "los pueblos de indios", colonización de la selva y distribución de la tierra). Pese a esos tímidos ensayos de diversificación temática, destaca la absoluta predominancia de estudios que procuran dar razón del conflicto zapatista desde diversos enfoques teóricos (lucha armada y guerrilla, organizaciones políticas, estado y democracia, indigenismo, identidad nacional y étnica) y 
desde diversas disciplinas, siendo las más socorridas la antropología por tradición y la ciencia política por la naturaleza de los hechos. Hay por ende una proliferación de datos y una plétora de producciones intelectuales o coyunturales sobre zapatismo en Chiapas, como movimiento social, como organización política o como paradigma de participación ciudadana.

Ese contexto obliga a quienes producen desde Chiapas y sobre Chiapas a situarse respecto de los debates, nacional e internacionales, sobre el zapatismo pero también respecto de las realidades sociales y políticas olvidadas de la región, desde su labor académica y muchas veces, desde sus compromisos personales. Por ende, implica que definan su ubicación en relación con las múltiples y contradictorias interpretaciones vigentes sobre el zapatismo, pero también respecto de interacciones que no pertenecen a esa esfera ni se inscriben en el marco ad hoc de reflexiones y de interpretaciones, constituido y afinado después del 94. De hecho, en las revistas, me llamó la atención el que haya habido artículos sobre las razones del conflicto desde 1994 y ulteriormente un número casi temático del Anuario del CESMECA. A la par, me interesó el que se haya mantenido constantemente un esfuerzo por dar cuenta de otros aspectos del contexto social, probablemente más pronunciado que en la producción de los investigadores que abordan Chiapas desde fuera y se abocan esencialmente a trabajar sobre las realidades reveladas por el zapatismo. Sin embargo, en Chiapas también, la fascinación que ejerce este movimiento, como prisma a través del cual leer e interpretar la realidad regional, provocó una reducción de los asuntos sociales analizados y nutrió una visión sesgada de cambios e inercias.

Ahora bien, para los centros de investigación interesados en situarse en los campos disciplina- rios que les corresponden y en asumir labores de difusión, la doble inscripción de la producción sobre movimiento social en el campo emergente sobre zapatismo y en otros más tradicionalmente atendidos recalca la necesidad de responder ciertos interrogantes: uno, ¿̇en qué medida es deseable que se desarrolle una línea de investigación regional sobre el tema del zapatismo, desde el lugar mismo en que surgió y se desarrolló, habida cuenta del carácter del fenómeno y de los efectos de moda en la producción intelectual? Dos, ¿`cuáles serían las estrategias adecuadas para que esa línea se inserte tanto en la tradición propia de investigación institucional como dentro del debate general, nacional e internacional sobre el fenómeno? Tres, ¿cuáles serían las condiciones que aseguren a los autores una repercusión académica en su ámbito disciplinario natural, a partir de sus centros de adscripción, no gracias a sus estrategias personales de movilidad?

Definir espacios de publicaciones centrados o en el análisis específico de una de las expresiones del movimiento social o en temáticas más transversales supone, en dado caso, contar con acciones comunicativas que rebasen la publicación de revistas anuales, semestrales o eventuales y que aseguren una difusión adecuada, en tiempo y en circulación, a sus autores. También implica una política editorial más acabada, en cuanto a los perfiles de las revistas, a la regularidad de su publicación, al papel de los comités editoriales, a los criterios para la aprobación de materiales, a la participación de autores procedentes de las instituciones propias y externas y a posición respecto del universo de las revistas académicas por una parte, en cuanto al tipo de productos deseables, a la estructura y al perfil de los números, a su presentación y a su radio de publicidad por la otra. 


\section{La producción académica sobre movimiento social en Chiapas, más allá del zapatismo}

Aunque la naturaleza y los enfoques de los productos de investigación dependan de factores múltiples y aunque, a su vez, la selección de los productos publicables esté ligada a las políticas de los centros y a las formas de colegiación de la vida académica, la coexistencia de tres revistas, con temas coincidentes y con autores compartidos, lleva a discutir la pertinencia del formato de cada una, en cuanto a estructura, a uso y a coexistencia. Sorprende el que los índices de los anuarios de estudios indígenas no estén recortados en diversos apartados sino presentados linealmente como una sucesión de artículos inconexos y el que el Anuario de la UNICACH tenga secciones, pero justificadas en la pertenencia disciplinaria más que en una diferenciación de los productos publicados conforme con su carácter (debates, opinión y artículos de fondo). Probablemente, por ser anuarios más que revistas (lo que las diferencia de la revista del CIHMECH en su segundo periodo), esas dos publicaciones no han separado sus rúbricas de tal manera que puedan acoger tanto productos académicos de investigación como intervenciones en el debate intelectual o incluso político, de índole menos académico.

Pasar de un modelo anuario a un modelo revista depende obviamente del interés y de la capacidad para llegar a acuerdos en el interior de los tres centros sobre la naturaleza y la función de las publicaciones, sobre su periodicidad, sobre el lugar que se desea ocupar en el campo académico, sobre su mercado de consumo, sus temáticas y sus autores. Los anuarios están generalmente pensados como instrumentos para socializar los productos internos de los investigadores, en círculos cerrados en torno a la especialización o a la región; las revistas entran en los espacios más amplios de la competencia académica y de la discusión intelectual. Cambiar de un formato a otro obligaría a definir los mecanismos de participación de quiénes escriben, los criterios de selección y las modalidades de aceptación de artículos (con selección de los productos recibidos según la propia productividad de los centros o conforme con invitaciones para los investigadores tanto de los centros que producen la revista como de otras instituciones en función de tópicos preseleccionados). Mientras no se precisen los nichos de impacto de las publicaciones y mientras no haya una mínima concertación sobre sus usos institucionales (para dar a conocer la producción de los centros) y disciplinarios (para situarse respecto de determinadas cuestiones teóricas o en relación con la dilucidación de problemas), será difícil saber si es mejor conservar o modificar el perfil de las publicaciones. Sin embargo, lo anterior conduce a formular una pregunta más, que a mí sólo me toca expresar: ¿es necesario mantener simultáneamente varias publicaciones, que se caracterizan por una circulación interna fuerte de los autores y por un recubrimiento parcial de sus perspectivas o sería conveniente negociar y aplicar una política editorial interinstitucional, que pueda permitir publicaciones conjuntas y una diversificación de los productos (libros, tesis, revistas), objetos de labores editoriales?

Las tres publicaciones comparten un rasgo común adicional, el del anclaje regional. En su mayoría, las indagaciones publicadas remiten a ciertas regiones de Chiapas, principalmente a los Altos, a la Selva y a las Cañadas y, en menor medida, al Soconusco (Alcalá, 1998, y Alcalá, 1995; Leyva y Ascencio, 1990). Los análisis concernientes a los espacios fronterizos son, en términos relativos, pocos (Villafuerte Solís, 1990, Hernández Cruz, 1990; González Ponciano, 
1990). Asimismo, son escasos los artículos que reflexionan sobre la idea de pertenencia a un espacio regional-el sur de México o la frontera- o sobre identidades locales o regionales (Gutiérrez Cruz, 1997; Preciado Coronado, 1996; Angulo Barreda, 1993; Pacheco Méndez, 1994). Hay sin embargo un esfuerzo por dar cuenta de algunos países de Centroamérica, patente sobre todo en el Anuario 1995 de la UNICACH donde se encuentran aportes sobre países como Honduras y Costa Rica (Barahona D., 1995; Barahona, 1995, y Argueta, 1995) y en trabajos que tratan de la movilidad internacional en Centroamérica (Ordoñez Morales, 1993). De hecho, la inclusión de algunos artículos que tratan, en otros contextos geográficos, asuntos de interés amplio muestra que la región no es un concepto que debería de limitar el estudio de los hechos al territorio propio e inmediato; la región debería ser también un espacio-pretexto para abordar temáticas transversales que interesan o a especialistas pertenecientes a otras disciplinas o a los académicos de otras entidades. Para ilustrarlo aludiendo otra vez a la situación actual, la cuestión indígena ha sido analizada en los últimos años, refiriéndola muchas veces a Chiapas aun cuando interesa a muchos otros estados. En los últimos años, en ciencias sociales, se ha mencionado reiterativamente los riesgos del localismo y las bondades de la transdiciplinariedad. No sé si la alternativa se plantea en forma tan dicotómica pero sí creo que siempre es preferible enfocar los problemas desde circunstancias diferentes y a partir de diversas perspectivas. Evidentemente, convocar a otros a reflexionar sobre los intereses propios a partir de circunstancias ajenas dependería por una parte de la constancia de un trabajo editorial planeado, por la otra de la inclusión condicionada de los autores institucionales en sus revistas propias. En particular, los comités editoriales habrían de tomar decisiones sobre la estructura deseable de las publicaciones, definiendo si son excluyentes respecto de la producción doméstica en función de tópicos seleccionados o abiertas forzosamente a los artículos que llegan.

Ahora, si dejamos de lado las preguntas que surgen de la interrelación, tal vez voluntariamente imprecisa, entre la producción académica regional y el acontecimiento que provocó el resurgimiento de Chiapas como objeto de análisis predilecto a partir de las ciencias sociales y nos abocamos a analizar los artículos sobre organización social, queda claro que éstos están esencialmente referidos a grupos indígenas, por el mismo perfil disciplinario de los centros que producen el Anuario de Estudios Indígenas y la revista del CIHMECH. Los actores del quehacer social están definidos más por su identidad étnica (choles, tzeltales, zoques, tzotziles) que por su condición social o por su régimen de acción.

Los procesos que están descritos se enmarcan en gran parte en esa definición étnica, independientemente de las otras identidades (genéricas, laborales) de los actores aludidos y de los diversos registros a los cuales remiten las prácticas y las formas de organización consideradas. En esa perspectiva, me parece que dos cuestiones son susceptibles de ser problemáticas a la larga: una es la de la elección como objetos de estudios de los actores que intervienen en los campos social y político así como de los niveles en los que se analiza su acción. Un repaso a los artículos revela una focalización sobre mujeres (Herrera Puente, 1999; Hernández Castillo, 1996; Perezgrovas, 1998; Fernández Guerrero, 1998; Santana Echeagarray, 1998; Garza Caligaris, 1990; Garza Caligaris, 1993) y, dentro de los estudios de género, sobre indígenas. Los núcleos a los cuales se refieren las formas de organización son la familia (Díaz de Salas, 1998; Rodríguez Galván y Zaragoza, 1998), la escuela, principalmente la indígena, bilingüe o 
bicultural (Medina, 1998, Freedson y Pérez, 1994, y Sulca, 1994; Padilla, 1987) y la religión (Calvo Sánchez, 1990, Hernández Castillo, 1991; Rivera Farfán, 1997 y Morquecho, 1996). Los enfoques más socorridos se centran en los modelos de organización políticas o sociales (Sonnleitner, 1998, Leyva y Ascencio, 1990; Harvey, 1991), en los compromisos políticos (Medina, 1995; Fábregas, 1993) y en los derechos humanos (Fernández Lira, 1995).

Otro punto que llama la atención es que, en la producción publicada por las revistas de ciencias sociales en Chiapas, las lógicas de funcionamiento del campo de la organización social o de la organización política están generalmente atendidas a través de las lógicas de los actores. Las indagaciones se proponen establecer los modelos categoriales de conducta más que identificar reglas grupales y códigos de interacción, con sus inercias y transformaciones. Hay mayor interés en describir o en analizar la manera cómo los actores se socializan en determinar estructuras que en dar cuenta de cómo tanto las políticas como las formas de movilización modifican las estructuras de los campos de acción y los espacios de actividad y de presencia pública de dichos actores. Hay a veces también una tendencia a concentrar la reflexión sobre situaciones sociales muy precisas y quizás, demasiado particulares.

Las excepciones a esas tendencias fuertes las representan los artículos que definen los actores en función de su inserción en la estructura productiva o laboral, como los profesionistas (Ascencio Franco, 1999), los pescadores y los chicleros (Arrivillaga, 1997). Por tema, son los que tratan la globalización en su relación con los esquemas de organización o de representación social (democracia -Villafuerte Solís, 1998) o con la ruralidad (Villafuerte Solís, 1996; Reyes Ramos, 1994)), las formas de organización y de control políticos (ONG en García Aguilar, 1997; ciudadanía en Guatemala, Sieder, 1997, Anuario; estado y sociedad, Arrivillaga, 1997), los problemas de seguridad (Ascencio Franco, 1996) y los patrones locales de desarrollo (Ascencio Cedillo, 1995; Whiting, 1994; Zárate e Hidalgo, 1993).

\section{Los pendientes}

No me corresponde hacer una valoración de los temas considerados respecto de los enfoques recientes sobre organización y movimiento sociales. La elección de determinados objetos o el cambio en los tópicos de investigación depende en extremo de las rutas de especialización de los centros de investigación y de sus tradiciones disciplinarias, de sus recursos, de su vida académica y de las decisiones personales de sus investigadores. Y, en muchas instituciones, el surgimiento, la consolidación o el debilitamiento de las áreas fuertes de investigación se produce más por casualidades, por relevos generacionales y por la posición de los grupos intelectuales en el campo que a raíz de recomendaciones sobre las tendencias de evolución de las disciplinas o de los campos de estudio.

Sin embargo, una clasificación de los artículos que remiten al eje de organización social en grandes categorías, sean esas amarradas al estudio de actores o de movimientos, sí me conducen a hacer algunos comentarios. En mi óptica personal, de alguien que dista de conocer a profundidad Chiapas, repito que son perturbadoras la intensa presencia de unos cuantos actores y la casi ausencia de otros muchos en los discursos de investigación. Me inquieta que esos actores estén analizados más en sí mismos que en sus transformaciones y en sus relaciones complejas, del antagonismo a la dependencia, con el Estado, con otros sujetos sociales y con instituciones u organizaciones. En la perspectiva de 
las interacciones obligadas, sean éstas basadas en la norma o en la violencia, aparece la escasez de estudios sobre los actores no indígenas, también esenciales si se pretende dar cuenta del funcionamiento rutinario de la sociedad y de sus dinámicas de cambio.

Por ende, creo indispensable dejar apuntado que los sectores modernos de la sociedad chiapaneca, los grupos sociales circulatorios, procedentes de otros lugares pero asentados en el territorio chiapaneco y las elites políticas, económicas, intelectuales y profesionales casi no han sido objetos de atención. Vale también insistir en que un desinterés similar pesa sobre los colectivos que impulsan una reinterpretación de los comportamientos, desde el ámbito de lo cotidiano hasta el de lo institucional, tengan esos formas visibles o no de organización y de movilización y tengan o no conciencia de su papel transformador respecto de prácticas sociales estáticas. Parece, no obstante, que la focalización creciente de las investigaciones sobre el indigenismo aplazó la posibilidad de consolidar otros ejes y niveles de análisis, igualmente importantes para comprender la acción social, como los de las políticas públicas y de las instituciones.

Por otra parte, en las coyunturas nacional e internacional, Chiapas es un espacio escindido, en donde se ejemplifican las sutiles interacciones entre tradición y transición y en donde surgen principios de estructuración conflictual o complementaria entre sujetos emergentes y sujetos convencionales. Probablemente, no es casual que muchos observadores de fuera insistan en el carácter innovador o atípico del zapatismo. Pero tampoco es un azar si se ha intentado explicar parte de la realidad chiapaneca gracias a la utilización de binomios como globalización-ruralidad, redes-clientelismo. Esos acercamientos revelan las correspondencias asincrónicas entre las tendencias que imperan en la coyuntura nacional y las que rigen en las escalas regionales y locales; muestran que producen desfases, rupturas y recomposiciones en las formas clásicas de la institucionalidad y de la vida pública que valdría la pena estudiar con más ahínco.

Tanto los indicadores estadísticos como los análisis académicos o las simples impresiones del viajero comprueban que Chiapas es un conglomerado de microrregiones tan diversas como es heterogénea y escindida su sociedad. Nunca es fácil rendir cuenta cabal de la diversidad, diversidad social y étnica en cada región y diversidad de las propias regiones en el mosaico estatal. Sin embargo, hay una necesidad, no cumplida a mi juicio, de explicar la unicidad territorial del estado y los dispositivos que explican la constitución de una ciudadanía chiapaneca, misma que rebasa e incluye las pertenencias locales. Esas dimensiones difícilmente aparecen en artículos con un sesgo localista pronunciado. Y allí haya tal vez un déficit de escala para pensar las lógicas de construcción de las identidades políticas, sociales y ciudadanas en la región.

Finalmente, recordemos que las palabras ni son inocuas, ni son neutras. Hay palabras que rondan en los discursos desde fuera sobre Chiapas como las de alteridad, de conflicto, de pluralidad y de gobernabilidad. Esas se declinan en Chiapas como racismo, violencia, exclusión y polarización. Los discursos paralelos expresan adscripciones disciplinarias y afiliaciones teóricas pero también intervenciones y posiciones políticas sobre las que no conviene abundar aquí pero sí señalar. Explican en parte el que, en Chiapas, encontremos una sociología del sujeto indígena más que una de la intersubjetividad, una sociología de la confrontación más que de las transacciones, una sociología de la pertenencia más que de las movilidades y una sociología de la 
marginación más que de la "potencia de actuar" para utilizar una expresión consagrada de Paul Ricoeur.

\section{Comentarios finales}

Para concluir esta intervención, quisiera retornar sobre la cuestión de las ciencias sociales como disciplina y retomar el asunto particular que nos reunió aquí, a saber, el de las revistas en Chiapas. Hace algunas semanas, consulté un documento preparado por un grupo perteneciente a la sección de ciencias sociales y humanidades de la Academia Mexicana de Ciencias, titulado "Propuesta para el desarrollo de las Ciencias Sociales y Humanidades en México" (http:/ / www.observatorio.org/amc.htm). Desde la introducción, el reporte define como sus objetivos centrales hacer un balance de las condiciones de desarrollo de las ciencias sociales y las humanidades en el país y emitir recomendaciones para elevar su calidad, su pertinencia y su repercusión social. Esos tres términos requieren contenidos precisos y discusiones, desde los lugares y las comunidades académicas que más directamente afectan. Su recurrencia, retórica o no, plantea centralmente la cuestión de los usos y de los destinatarios del conocimiento producido por las ciencias sociales, más allá del ámbito académico de los pares disciplinarios; sobre todo, obliga a definir lo que se entiende por esas nociones, tan frecuentes y tan vagas, de conocimiento útil, de conocimiento estratégico y de conocimiento de frontera. Más todavía que precisar temáticas, lo que urge es discutir las contribuciones de la disciplina a la comprensión del entorno, tomando en cuenta las condiciones de la producción académica y las del contexto. Eso es cuanto más crucial que, en el debate cultural, están en disputa un discurso transversal sobre la función de las ciencias sociales en la sociedad del conocimiento, en una óptica generalizante de enriquecimiento de los saberes, por una parte y, por la otra, discursos disciplinarios que abogan por la indigenización de las ciencias sociales y redefinen su pertinencia en la perspectiva regional de su aplicación.

\section{Bibliografía}

Alcalá, G., "Una aproximación al desastre: La modernización impuesta a los pescadores de El Soconusco", Anuario 1995, Centro de Estudios Superiores de México y Centroamérica/ Universidad de Ciencias y Artes del Estado de Chiapas, pp. 125-153, México.

Angulo Barredo, J.I., 1996, "Algunas consideraciones sobre cultura, economía y migración en Los Altos de Chiapas", Anuario IEI, Instituto de Estudios Indígenas/Universidad Autónoma de Chiapas, Núm. vi, pp. 161-176, México.

—, 1995, "Comportamiento de la población regional y grupos étnicos en los altos de Chiapas” (1960-1990), Anuario IEI, Instituto de Estudios Indígenas/Universidad Autónoma de Chiapas, Núm. v, pp. 193-208, México.

—, 1994, "Población y migraciones campesino-indígenas de Los Altos de Chiapas", Anuario IEI, Instituto de Estudios Indígenas/Universidad Autónoma de Chiapas, Núm. Iv, pp. 43-56 México.

Aparicio, J.A., "Malinalco, Estado de México. Notas sobre población y dinámica demográfica", Anuario 1997, Centro de Estudios Superiores de México y Centroamérica/ Universidad de Ciencias y Artes de Chiapas, pp. 433-455, México.

Aramoni Calderón, D. y Morquecho Escamilla, G., "La otra mejilla... pero armada. El recurso de las armas en manos de los expulsados de San Juan Chamula", Anuario 1996, Centro de Estudios Superiores de México y Centroamérica/Universidad de Ciencias y Artes del Estado de Chiapas, pp. 533-552, México. Argueta, M.R., "Tres momentos en la conformación de la identidad nacional hondureña", Anuario 1995, Centro de Estudios Superiores de México y Centroamérica/Universidad de Ciencias y Artes del Estado de Chiapas, pp. 326-334, México.

Arrivillaga Cortés, A., "Chicle, chicleros y chiclería. Sobre su historia en El Petén”, Anuario 1996, Centro de Estudios Superiores de México y Centroamérica/Universidad de Ciencias y Artes del Estado de Chiapas, pp. 362-398, México.

- "El Caribe centroamericano, una visión regional y política", Anuario 1999, Centro de Estudios Superiores de México y 
Centroamérica/Universidad de Ciencias y Artes de Chiapas, pp. 243-270, México.

Baek L., Text and context in the thematization on development, uNESCO, International Bureau on Education, Prospects, Vol. Xxxix, Núm. 4, december 1999, pp. 459-480, Bélgica.

Barahona, M., "Identidad, estado e integración nacional: el caso de Honduras", Anuario 1995, Centro de Estudios Superiores de México y Centroamérica/Universidad de Ciencias y Artes del Estado de Chiapas, pp. 292-325, México.

Barjau, L., "No somos mestizos", Centro de Investigaciones Humanísticas de Mesoamérica y el Estado de Chiapas/ Universidad Nacional Autónoma de México, СІнмесH, Vol. 5, Núm. 1 y 2, enero-diciembre 1995, pp. 47-58, México.

Bématouil T., 1999, Critique et pragmatique en sociologie, Ecoles des Hautes Etudes en Sciences Sociales, Annales, Núm. 2, 281-317, París.

Burguete Cal y Mayor, A., 1998, "Procesos autonómicos indígenas en la región Altos de Chiapas", Anuario de Estudios Indigenas, Instituto de Estudios Indígenas/Universidad Autónoma de Chiapas, Núm. vII, pp. 127-158, México.

—, "Sistemas normativos indígenas para la regulación del agua en Chamula y Zinacantán”, Anuario 1998, Centro de Estudios Superiores de México y Centroamérica/Universidad de Ciencias y Artes del Estado de Chiapas, pp. 233-263, México.

Calvo-Sánchez, A., 1991, "Las colonias nuevas de migrantes y expulsados en San Cristóbal de Las Casas", Anuario CEI III, Centro de Estudios Indígenas/ Universidad Autónoma de Chiapas, pp. 55-64, México.

Fábregas Puig, A., "Una reflexión sobre el conflicto chiapaneco", Anuario 1994, Departamento de Patrimonio Cultural e Investigación/Instituto Chiapaneco de Cultura, pp. 9-20, México.

Fernández Guerrero, M. y Freyermuth-Enciso, G., 1996, "Mujeres indígenas y derechos reproductivos, el caso de las mujeres de San Juan Chamula", Anuario IEI, Instituto de Estudios Indígenas/ Universidad Autónoma de Chiapas, Núm. vi, pp. 177-192, México.

Fernández Lira, C., 1995, “Identidad y razón en Chiapas. Algunas reflexiones sobre la cuestión de los derechos humanos", Anuario IEI, Instituto de Estudios Indígenas/Universidad Autónoma de Chiapas, Núm. v, pp. 91-110, México.

Ferrera C, N., 1998, "Estrategias de reproducción socieconómica en las comunidades indígenas de los Altos de Chiapas", Anuario de Estudios Indigenas, Instituto de Estudios Indígenas/
Universidad Autónoma de Chiapas, Núm. vII, pp. 309-334, México.

Franco, R.A., "Los profesionistas y el juego del conocimiento", Anuario 1999, Centro de Estudios Superiores de México y Centroamérica/ Universidad de Ciencias y Artes de Chiapas, pp. 116-141, México.

Gall, O., "El racismo en el contexto de realidades nacionales y regionales diversas: discusión acerca de una propuesta teórica de análisis", Anuario 1996, Centro de Estudios Superiores de México y Centroamérica/Universidad de Ciencias y Artes del Estado de Chiapas, pp. 160-208, México.

García Aguilar, M.C. y López-Meza, A., "La Acción Institucional y Su Impacto en los Altos de Chiapas", Anuario de cultura e investigación 1990, Instituto Chiapaneco de Cultura/ Departamento de patrimonio cultural, pp. 188-203, México. García Méndez, J.A., "La colonización chol de la selva: la fundación de Frontera Corozal", Anuario 1999, Centro de Estudios Superiores de México y Centroamérica/Universidad de Ciencias y Artes de Chiapas, pp. 183-203, México.

Garza Caligaris, A.M., 1991, "Sobre mujeres indígenas y su historia", Anuario CEI III, Centro de Estudios Indígenas/ Universidad Autónoma de Chiapas, pp. 31-42, México.

—, y Cárdenas Gordillo, B., 1994, "Anticoncepción y derechos reproductivos en Los Altos de Chiapas”, Anuario IEI, Instituto de Estudios Indígenas/Universidad Autónoma de Chiapas, Núm. IV, pp. 93-100, México.

González Ponciano, J.R., "Identidad y carácter nacional en Mesoamérica", Anuario 1993, Departamento de Patrimonio Cultural e Investigación/Instituto Chiapaneco de Cultura, pp. 150-183, México.

Harvey, N., 1991, "Estrategias corporativistas y respuestas populares en el México rural: Estado y organizaciones campesinas en Chiapas desde 1970", Centro de Investigaciones Humanísticas de Mesoamérica y el Estado de Chiapas/ Universidad Nacional Autónoma de México/Instituto de Investigaciones Históricas, СIHMECH, Vol. 2, pp. 51-66, México. Hernández Castillo, R.A., 1991, "Cambio y reelaboración religiosa: los testigos de Jehová en una comunidad chuj-k’anjobal de Chiapas", Anuario CEI III, Centro de Estudios Indígenas/ Universidad Autónoma de Chiapas, pp. 113-126, México.

Hernández Cruz, O., "El Caribe costarricense: dinámica regional y configuración multicultural de las identidades", Anuario 1997, Centro de Estudios Superiores de México y Centroamérica/ Universidad de Ciencias y Artes de Chiapas, pp. 210-253, México. 
Herrera Puente, M.E. "Mujercitas tzotziles y tzeltales: El precio de la emancipación femenina en Los Altos de Chiapas", Anuario 1999, Centro de Estudios Superiores de México y Centroamérica/ Universidad de Ciencias y Artes de Chiapas, pp. 284-306, México. Kauffer Michel, E.F., 1998, "Los refugiados guatemaltecos en Chiapas y los Derechos Humanos. De la búsqueda de la seguridad a la organización política", Anuario de Estudios Indigenas, Instituto de Estudios Indígenas/Universidad Autónoma de Chiapas, Núm. vII, pp. 283-308, México.

Lee Whiting, T.A., "Probabilidades de expansión del turismo arqueológico y la demanda de servicios turísticos”, Anuario 1994, Centro de Estudios Superiores de México y Centroamérica/ Universidad de Ciencias y Artes del Estado de Chiapas, pp. 516-534, México.

Leyva Solano, X. y Ascencio-Franco, G., "Espacio y Organización Social en la Selva Lacandona: El Caso de la Subregión Cañadas", Anuario de cultura e investigación 1990, Instituto Chiapaneco/Departamento de patrimonio cultural, pp. 17-49, México.

Lisbona Guillén, M., "A orillas del conflicto neozapatista: cavilaciones en torno a la construcción del Estado", Anuario 1998, Centro de Estudios Superiores de México y Centroamérica/Universidad de Ciencias y Artes del Estado de Chiapas, pp. 9-100, México.

Medina, A., "El zapatismo chiapaneco y la figura nacional del indio en México", Anuario 1995, Centro de Estudios Superiores de México y Centroamérica/Universidad de Ciencias y Artes del Estado de Chiapas, pp. 9-35, México.

Medina, F.X., "Consideraciones sobre las migraciones vascas a América. Apuntes sobre el caso de México”, Anuario 1996, Centro de Estudios Superiores de México y Centroamérica/ Universidad de Ciencias y Artes del Estado de Chiapas, pp. 310-325, México.

Moscoso Pastrana, P., "Los convenios de Esquipulas”, СIHMECH. Centro de Investigaciones Humanísticas de Mesoamérica y el Estado de Chiapas/ Universidad Nacional Autónoma de México/Instituto de Investigaciones Históricas, CIHMECH 1987, pp. 77-86, México.

Orantes García, J.R., 1994, “Contribución al estudio de la(s) identidad(es) urbana(s): variaciones de una leyenda", Anuario IEI, Instituto de Estudios Indígenas/Universidad Autónoma de Chiapas, Núm. Iv, pp. 57-66, México.

Ordóñez Morales, C.E., "Braceros chiapanecos y guatemaltecos en un mercado de trabajo regional", Centro de Investigaciones
Humanísticas de Mesoamérica y el Estado de Chiapas/ Universidad Nacional Autónoma de México, CIHMECH, Vol. 3, Núm. 1, enero-junio 1993, pp. 17-34, México.

Pacheco Méndez, T., "Chiapas en el contexto del proyecto modernizador”, Centro de Investigaciones Humanísticas de Mesoamérica y el Estado de Chiapas/Universidad Nacional Autónoma de México, CIHMECH, Vol. 3, Núm. 2, julio-diciembre 1993, pp. 47-58, México.

Paniagua Mijangos, J., 1991, "Movimientos de población en la Sierra Madre: orígenes sociales", Anuario CIE III, Centro de Estudios Indígenas/Universidad Autónoma de Chiapas, pp. 215226, México.

Perezgrovas Garza, Raúl, 1998, “Colaborando para el cambio. Pastoras tzotziles de Chiapas participan en el diseño de estrategias de desarrollo", Anuario de Estudios Indígenas, Instituto de Estudios Indígenas/Universidad Autónoma de Chiapas, Núm. vII, pp. 347-370, México.

Preciado Coronado, J., "Chiapas y América Latina”, Anuario 1996, Centro de Estudios Superiores de México y Centroamérica/Universidad de Ciencias y Artes del Estado de Chiapas, pp. 243-303, México.

Reyes Ramos, M.E., "Colonización de tierras en Chiapas", Centro de Investigaciones Humanísticas de Mesoamérica y el Estado de Chiapas/Universidad Nacional Autónoma de México, СІнмеCH, Vol. 4, Núm. 1 y 2, enero-diciembre 1994, pp. 53-72, México.

Rivera Farfán, C., "La política de los cargueros. Los cargueros de la política. El Cabildo en una localidad purhépecha", Anuario 1998, Centro de Estudios Superiores de México y Centroamérica/Universidad de Ciencias y Artes del Estado de Chiapas, pp. 194-209, México.

Rodríguez Galván, G. y Zaragoza Martínez, L., 1998, "Organización y división del trabajo en la unidad familiar de la Sierra Madre de Chiapas", Anuario de Estudios Indígenas, Instituto de Estudios Indígenas/ Universidad Autónoma de Chiapas, Núm. viI, pp. 335-346, México.

Supiot A. et. alt. Premier rapport annuel d' activités. Francia, Ministère de $1^{‘}$ Education Nationale, de la Recherche et de la technologie/Conseil National du développement des sciences humaines et sociales, junio 1999.

Van der Haar, G., 1998, "El fin de las fincas comitecas de la zona alta tojolabal: historia de un desenlace", Anuario de Estudios Indígenas, Instituto de Estudios Indígenas/Universidad Autónoma de Chiapas, Núm. VII, pp. 105-126, México. 
Villafuerte Solís, D., "Frontera sur y globalización”, Anuario 1997, Centro de Estudios Superiores de México y Centroamérica/Universidad de Ciencias y Artes de Chiapas, pp. 9-36, México.

—, "Globalización, neoliberalismo y democracia. Notas para una lectura desde la periferia", Anuario 1998, Centro de Estudios Superiores de México y Centroamérica/Universidad de Ciencias y Artes del Estado de Chiapas, pp. 101-127, México.

—, "La ruralidad en tiempos de la globalización: problemas y enfoques", Anuario 1996, Centro de Estudios Superiores de México y Centroamérica/Universidad de Ciencias y Artes del Estado de Chiapas, pp. 119-159, México.

—, y García Aguilar, M.C., "A propósito de la insurrección zapatista. Notas sobre economía y sociedad en Chiapas, México", Anuario 1995, Centro de Estudios Superiores de México y Centroamérica./Universidad de Ciencias y Artes del Estado de Chiapas, pp. 36-74, México.

-, y García Aguilar, M.C. "Los empresarios cafetaleros de Soconusco ante la crisis", Anuario 1993, Departamento de Patrimonio Cultural e Investigación/Instituto Chiapaneco de Cultura, pp. 318-341.
Villasana Benítez, S., 1994, "Los zoques de Chiapas. Su distribución demográfica en 1990”, Anuario IEI, Instituto de Estudios Indígenas/Universidad Autónoma de Chiapas, Núm. IV, pp. 203-230, México.

Villasana Benítez, S. y Martínez Mendoza, A., 1998, "La educación indígena en Chiapas. Experiencias de los tzeltales durante la década de 1950", Anuario de Estudios Indígenas, Instituto de Estudios Indígenas/Universidad Autónoma de Chiapas, Núm. vII, pp. 227-264, México.

Zárate Vargas, G. e Hidalgo-Pérez, M., "Economía de la pobreza: Los jornaleros de los Altos de Chiapas", Centro de Investigaciones Humanísticas de Mesoamérica y el Estado de Chiapas/Universidad Nacional Autónoma de México, CIHMECH, Vol. 3, Núm. 1, enero-junio 1993, pp. 165-184, México.

Ziccardi A. y R. Rodríguez (coord.), Propuesta para el desarrollo de las Ciencias Sociales y Humanidades en México (documento para la discusión), Academia Mexicana de Ciencias, Sección de Ciencias Sociales y Humanidades, México, marzo 2001 en http:// www.observatorio.org/amc.htm 